\title{
Quality Aspects in Production of Electronic Grade Chemicals in Multipurpose Plants
}

\author{
Xenia Beyrich-Grafa* and Guy Seltensperger ${ }^{\mathrm{b}}$
}

\begin{abstract}
Electronic devices and displays require electronic grade chemicals for their manufacturing process. As miniaturisation in the electronics industry has pushed the boundaries of physics, no contamination can be tolerated in the production of microchips or display panels. Electronic grade chemicals need to be of highest purity and quality consistency. After giving an introduction to the quality requirements in the electronics industry, this article describes the path from laboratory synthesis to a capable production process of a small volume electronic specialty material in a multipurpose plant. The upscaling of an electronic chemical for electronic device manufacturing is one of the examples of how products with high quality requirements can be successfully produced in an existing production infrastructure.
\end{abstract}

Keywords: Electronic chemicals · Multipurpose plant · Process optimisation · Statistical process control

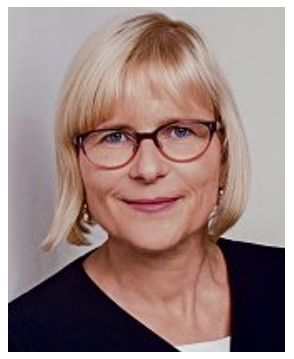

\section{Introduction to Electronic Grade Chemicals}

Chemicals and materials required for manufacturing and packaging semiconductors, and high-density circuits are commonly called 'electronic chemicals'. More than 100 different products are used in these applications and some of the typical product categories are acids and etchants, gases, cleaners, solvents, photoresists and liquid crystals, only to name a few. ${ }^{[1]}$ BASF is also selling electronic chemicals to the semiconductor industry. The product portfolio includes acids, gases and solvents as well as formulations for advanced cleaning, etching, ancillaries for photoresists, wet deposition and plating solutions.

A semiconductor device can be described as an electronic circuit comprising semiconducting material such as doped silicon that can be used to control electric charge, current and voltage for switching, amplification and energy conversion. Discrete semiconductor devices perform one single elementary function, i.e. electrical switching or rectification. Integrated circuits (IC) combine multiple electronic functions on one common semiconductor substrate. Since the invention of the transistor in 1947 and the development of the integrated circuit in 1958, the semiconductor business has grown rapidly. Semiconducting materials, such as silicon and gallium arsenide are produced as monocrystalline solids and are cut into thin circular slices or wafers to serve as the substrate in manufacturing integrated circuits, for example microprocessor chips. ${ }^{[1]}$

\subsection{Moore's Law}

Chips make up the processing and memory units of the modern digital computer. Since the 1960s the number of integrated circuits per chip has grown enormously. In 1965 Gordon Moore, one of the co-founders of Intel, predicted that the number of transistors on a chip will double every two years. This principle also known as Moore's law describes the development from a few thousand transistors per chip in 1971 (Intel Corp.'s first chip) to more than twenty billion in 2016. Due to the huge shrinkage of the transistor dimensions from micrometres to nanometres chip making is performed in a 'clean room', (Fig. 1) since even microscopic contaminations could render a chip defective. ${ }^{[2]}$

\subsection{Quality Requirements for the Electronic Industry}

As the microchip production involves many different chemicals and more than 1000 process steps, all employed chemicals must be of exceptional purity to prevent failures. The electronic industry imposes strict quality standards on their chemical suppliers. The required purity for cleaning chemicals in the chip production ranges from 20-100 ppb for SSI chips (small-scale integration, 10 transistors per chip) to less than $10 \mathrm{ppt}$ for SLSI chips (super-large-scale integration, 1-10 million transistors per chip).

In order to produce electronic chemicals at consistent high quality, the chemical manufacturers need to maintain a robust process control system, for which all operators are trained and methods of statistical process control (SPC) are validated. The manufacturer shall apply a production 
process which within its normal statistical variations can consistently deliver the required product quality. This is usually described as process capability. Quality parameters are monitored with process control charts like the example shown in Fig. 2. In addition to the Upper Specification Limit (USL) and the Lower Specification Limit (LSL) a control chart contains an Upper Control Limit (UCL) and a Lower Control Limit (LCL). The distance of the UCL and the LCL from the process average (mean) is the triple standard deviation $\sigma$ of the process.

The capability of a process (Cpk) is calculated by the following Eqn. (1). In a capable process, the UCL and LCL are well within the USL and LSL resulting in a Cpk of $>1$. Semiconductor manufacturers usually expect process capabilities with a Cpk of $>1.3 .{ }^{[3]}$

$$
C p k=\min \left(\frac{\mu-L S L}{3 \sigma} ; \frac{U S L-\mu}{3 \sigma}\right)
$$

\section{Fast Scale-up of an Electronic Chemical}

The electronic chemical described in this article is made in a multistep synthesis. The lab process was transferred from the research labs of Ciba Specialty Chemicals, since 2009 part of BASF, to the process development labs in Schweizerhalle, where the different steps were scaled up to pilot scale in only five months. In 2003 the process was first produced in the pilot plant, in a special reactor setup dedicated for electronic specialties. After the successful pilot production of $350 \mathrm{~kg}$, the process development team focused on developing the production process. Based on the limited experience of only one pilot campaign, the team had the challenge to quickly define a production process, that would lead to the required product quality at acceptable yields. As the stringent quality requirements of the display manufacturers require a requalification process for any process changes, the process conditions would be frozen for the years to come.

\subsection{Launch Platform Concept}

It was planned to directly introduce the product into the multipurpose production unit WS-2049 in Schweizerhalle. This production unit is part of the so-called launch platform in Schweizerhalle. The launch platform consists of process development labs, kilo lab, pilot facilities, multipurpose production and process analytics all on one site. This concept allows the fast transfer of specialty products from process development to production (Fig. 3). The launch platform is especially suited for electronic

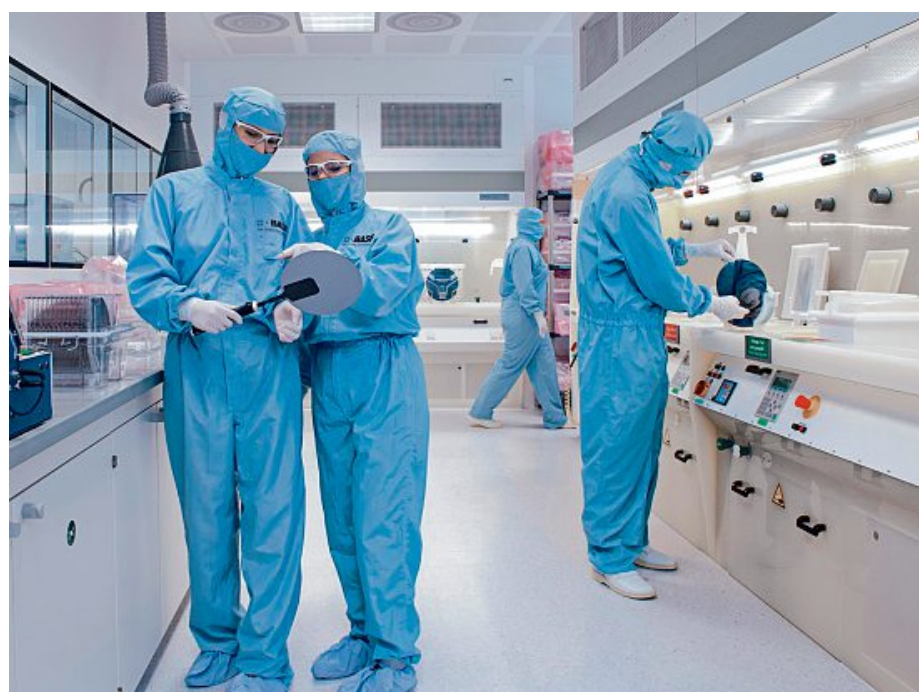

Fig. 1. Chip manufacturing requires high purity chemicals. Production and analytics is performed in a 'clean room'. The above picture shows a BASF test lab for cleaning chemicals used for silicon wafers.

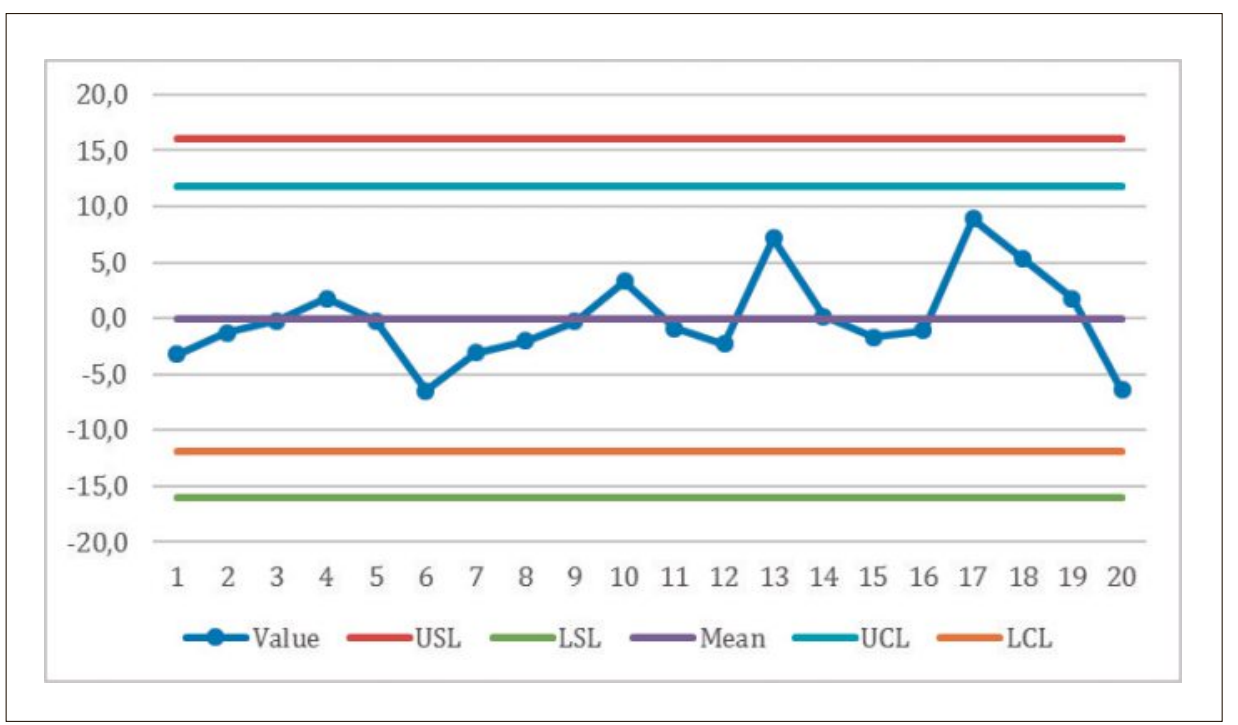

Fig. 2. SPC Chart of a capable production process with $\mathrm{Cpk}=1.35$.

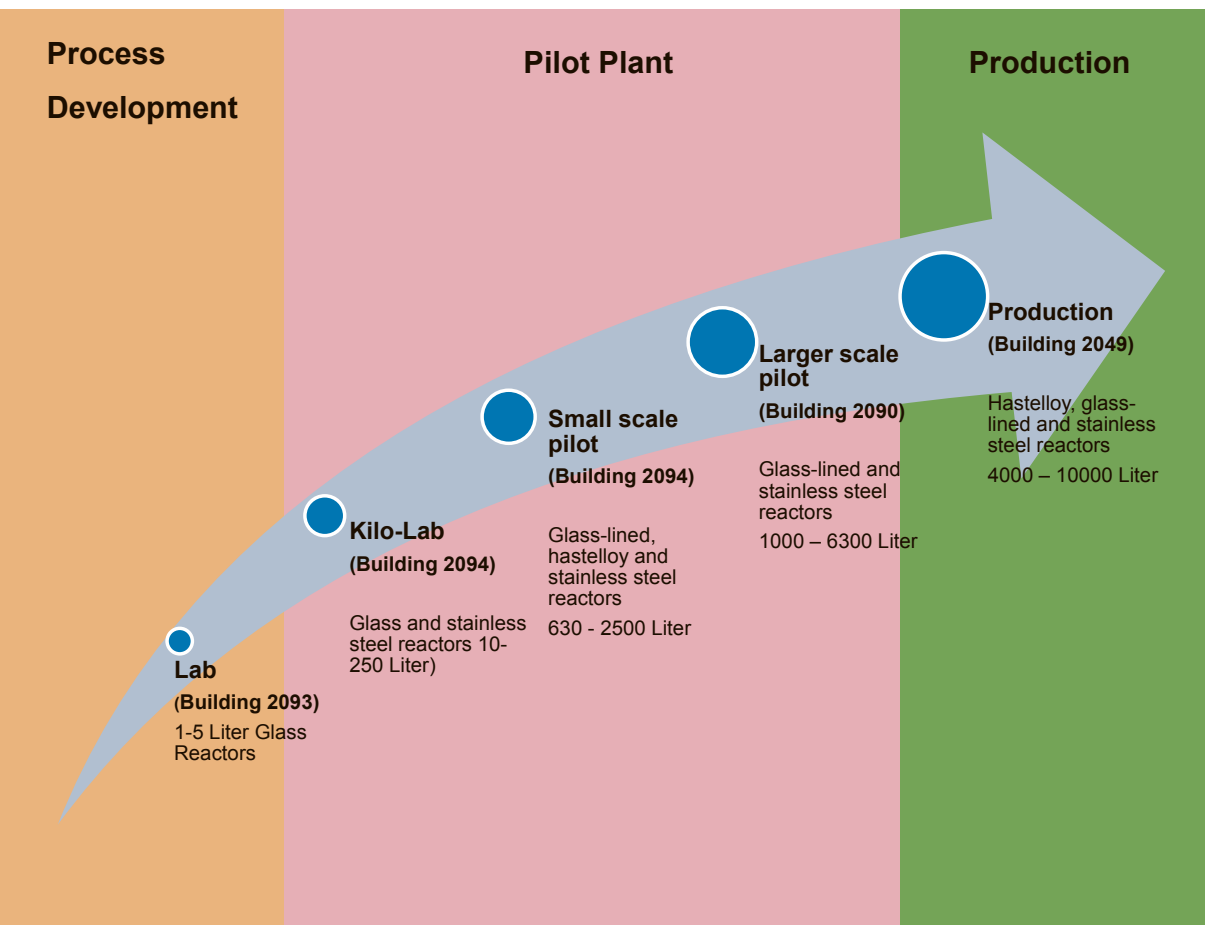

Fig. 3. Launch Platform Concept for fast introduction of new specialty products in Schweizerhalle. 
specialties, as Schweizerhalle is equipped with an ultrapure water unit and many reactors are glass-lined. These are important prerequisites to meet metal ion specifications in the ppm and $\mathrm{ppb}$ range.

Building 2049, the biggest plant in the launch platform, is specialized in campaign production of launch quantities or smaller products (Fig. 4). There are no dedicated production lines. The unit has a highly flexible setup allowing a quick changeover between products with an annual production capability of over 25 different isolated intermediates and products.

The process development was successful and in summer 2004 the multistep process was introduced in building 2049 and the product could be sold. Making commercial quantities of a recently developed product in such a short time was a joint effort of team members from research, process development, production and marketing.

\subsection{Process Optimisation and Quality Improvement}

During the next years the electronic chemical was produced in campaigns to satisfy the growing market demand. Customers asked for additional quality parameters. Whereas the product specification in the first campaigns contained 10 test parameters, it has grown to 19 parameters over time. Fluctuations in raw material quality and sensitivity to changes in some process conditions led to quality variations and occasional off-spec material, which needed to be recrystallised. The data shown on the SPC chart in Fig. 5 demonstrate that although in the first three campaigns the process mean is well above the specification value, the variances are
Fig. 4. Multipurpose Production Building WS-2049 in Schweizerhalle.
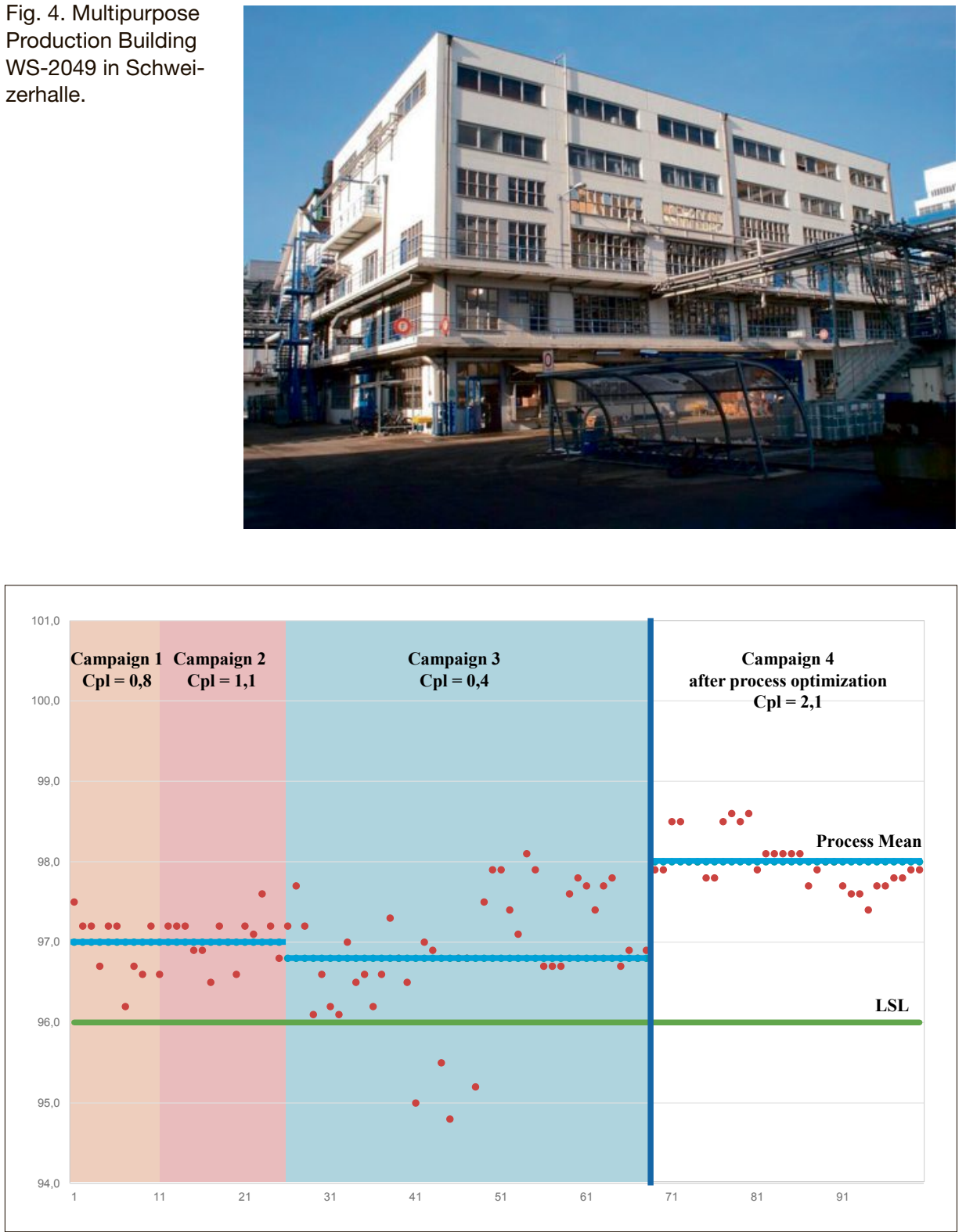

Fig. 5. SPC chart of one of the quality parameters in the production process. Process improvements lead to a significant increase of the process capability index Cpl. too high, resulting in a process capability index $<1$. As electronic customers require a capable process, it became apparent that process improvements had to be implemented.

Based on the data of the last production campaigns, all steps of the process were optimized starting from the raw material. This resulted in incremental but effective changes of reaction temperatures, temperature ramps, reagent stoichiometry and reaction times. After successful test batches and requalification by the customers, the optimised process was reintroduced into production in building 2049.

To stay on top with the technical progress in the electronics industry, two new next generation products have been designed with improved properties compared to the first product. Both have been introduced to the market according to the successful launch concept of the first product.

\section{Conclusion}

The manufacturing process of electronic chemicals needs to be reliable and lead to products of high purity and quality consistency. The fast technical developments of the electronic industry calls for new chemical solutions. In order to be able to bring these products to the market fast, an efficient concept is required that is able to deliver product and quality in time. In the production of electronic specialties constant process control and process optimisation is necessary to maintain the increasing customer requirements.

\section{Acknowledgements}

The authors thank all the colleagues and all their coworkers who have performed the work that is presented here in this article, especially Alain Wolf and Christian Schregenberger.

Received: February 6, 2018

[1] S. Daigle, E. Vogelsberg, B. Lim, I. Butcher, 'Electronic Chemicals' in 'Ullmann's Encyclopedia of Industrial Chemistry', WileyVCH, Weinheim, 2012

[2] a) https://www.britannica.com/technology/computer-chip/, accessed on January 5, 2018; b) M Waldrop, Nature 2016, 530, 144.

[3] J. S. Oakland, 'Statistical Process Control', Routledge, 2011. 